\title{
Reducing the Data Rate in Internet of Things Applications by Using Wireless Sensor Network
}

\author{
https://doi.org/10.3991/ijoe.v16i03.13021 \\ Omar Hashim Yahya \\ Northern Technical University, Mosul, Iraq \\ Haider Th. Salim ALRikabi $\left({ }^{凶}\right)$ \\ Wasit University, Wasit, Iraq \\ hdhiyab@uowasit.edu.iq \\ Ibtisam A. Aljazaery \\ Babylon University, Babylon, Iraq
}

\begin{abstract}
Due to the raising number of using Internet of Things (I O T) for personal and commercial devices and applications, as well as the continues needs for improving the quality of the performance for the internet - connected devices enhance the researchers to investigate solutions for managing the data through the network Because of the fact that the internet-connected devices needs addresses for each sensor node uses the IP-network and a large number of electronic modules is fabricated to be compatible with IPv4 only like Node Mcu, ESP8266, Arduino WIFI, and ESP32 with knowing that the IPv4 has a limited addresses number that will not be enough for the needs of IOT developments, Many researchers turned to IPv6. In the proposed system, another solution is suggested instead of the IPV6. The proposed idea is based on Non-IP Wireless Sensor Network (WSN) by connecting many sensing nodes to the sink-node that has an IP to forward the data to the server to be visualized in a web-portal. By this method, it is able to connect more than one node to the internet over only one IP, there for the data rate that are needed will be decreased. In addition, by using the non-IP network, the data rate and the power consuming by the sensor nodes have been reduced, the practical results are discussed for connecting four nodes over one IP4 and how it will reduce the data rate and the power consuming. In this work, the esp33 has been used as a Sink-node, and the wireless transceiver module ( NRF24) has been utilized to transmit the data from nodes to esp32.
\end{abstract}

Keywords - Internet of Things (IOT), Wireless Sensor Network (WSN), Data Collection and Data Visualization.

\section{Introduction}

The Wireless sensor network (WSN) is a general term to describe a set of tiny computers with the main purpose of detecting some physical property like tempera- 
ture, humidity, vibration, current, voltage, and energy or power [1,2]. They consist of many sensor nodes. Typically, there is at least one special node called the base station or the sink-node, which connects the network or sensors to the outside world. These sensor nodes are connected either to other nodes, or to the sink-node directly, this connection is done via one of the wireless communication techniques.

These nodes have a limited power source, sensing, processing, and communication abilities. Most importantly is the limitation of the power source. Because in many applications if the sensor node is fully exhausted its energy, it is impossible to replace it[3]. Therefore, the energy consumption of the sensor nodes is the most challenging limitation for the long-run operation of WSNs. Different cases have been researched for this purpose and included energy-aware medium access control (MAC) layer protocols, duty-cycling and low-power radio frequency communication hardware.

In recent years, the device-to-device communication or device to human communication has played an important role. These devices are used for industrial applications, smart vehicles, home appliances, personal electronics, and health monitoring [4-6]. The help of internet connectivity with the use of these devices derives the platform that known as the Internet of Things (IOT). Almost, all the devices which are used for home appliance, industrial automation devices, health monitoring, and vehicles have increased the adoption of the IOT [7]. The low power wireless personal area networks (6LoWPAN) play an important role in the IOT implementation because the TCP/IP has some limitations in achieving device-to-device communication. 6LoWPAN has become a standard communication technology for device-to-device in the IOT. Much research has been carried out on the use of WSNs in the IOT.

The Internet of Things is a great development in information technology (IT) domain, and is a result of the multiple technologies convergence, like embedded systems, machine learning, real-time analytics and commodity sensors [8-10].

\section{Literature Review and Problem Statement}

There are many researches and related to WSN and the way to implement the IOT systems. For example, the authors [11] proposed a WSN for observation frameworks in home, office, or plant condition by using PIR motion sensors. The system that presented here mainly has three different parts: various PIR sensor hubs that are conveyed into intrigued space or room and send its PIR sensor data to the base hub, the base hub (which is associated with the base station) gets/advances sensor data, and the base station workstation which assembles sensor data and performs the locale based following calculation. The outcome is shown on the screen of the personal computer (PC). This system uses ZigBee technology as a wireless model to transmit data.

While in [12] the proposed idea is developing a remote monitoring system for the smart home. This system combined embedded technique with Global System for Mobile communications (GSM). It contains Liod platform main controller, microcontroller module, GSM module, Universal Serial Bus (USB) camera and sensors (smoke, gas leak and infrared). GSM module was communicated to transmit all the information gathered by this system. 
The system proposed in [13] produced a remote home security system by combining the advantages of the GSM technology with the WSN. It is able to detect the fire and the intrusion and notify the user about the incidence remotely. Based on combining wireless communication and sensors, this system is able to detect, collect, and process the information of the object in the area that it covered. Then, transmit the data to the observer mobile by using GSM. This system used ZigBee technology as a wireless model to transmit data in WSN.

The authors in [14] have developed a portable data acquisition system (PANDA) based on Shinyei PPD42NS and had data processed on the server. These systems were described in their work; all performed accurately after proper calibration and revealed their pragmatic value in participatory or distributed sensing. The authors developed a battery-operated aerosol-monitoring tool by using low-cost shelf optical aerosol sensors. And based on this tool, they made a report on the field calibrations. In addition, by using the method of the federal equivalent for the $24 \mathrm{~h}$ and $1 \mathrm{~h}$ PM2.5 data class III US EPA, their instrument was calibrated. With ambient temperature and humidity, a negligible association was observed. In the results, it was found that $60 \%$ of the variance in $1 \mathrm{~h}$ reference PM2.5 data and $72 \%$ of the variance in $24 \mathrm{~h}$ data have been explained by the linear correction. The $60 \%$ performance $1 \mathrm{~h}$ can be compared to the instruments that commercially available and more costly.

The authors [15] proposed a monitoring system that based on IOT technology; they used a tri-level context for making the service of the context-aware, and designed it using an open source software and hardware. The testing was done using two scenarios as a smart home that based on IMS. The service of the disaster management is the first one which let the user be notified when specific situations occurred, like gas leakage, fires and break-ins. The service of the health care for the home is the second scenario, like the management of the deep sleep and a comfortable management for the house.

One of studies designed a system that consists of WSN and Server to analyze the data that collected from the network [16]. The Self-adaptive Kalman Filter (SAKF) was used in this system which is able to detect multiple environmental factors, collect data from multiple nodes, and then the collected data will be visualized in real-time. Each node is able to sense multiple factors, including UV radiation, atmospheric pressure, PM2.5, humidity, temperature, and geographical location. Each node data are transmitted to the server via WIFI networks to be stored in the database.

Another method [17] presented a work of a wireless sensor network for solar photovoltaic (PV) plant. It is an IOT objective solution for a Monitoring System. This system displays a remote energy performance and its yields on smartphones or on a computer to test the concept. The presented system supported with eight solar panels for monitoring temperature, humidity, string Current, and string voltage. Wi-Fi communication technique enhances the performance of the system with reduced area and eases the monitoring system performance after each 30 second. This proposed system used CC3200 microcontroller with ARM Cortex-M4 as a core.

In [18] the authors design and implementation smart method for register the Attendance of student by using Arduino to save efforts and time. 
In [19], the authors designed a reconfigurable interface with smart sensor for the industrial WSN in IOT systems. The system was able to collect the data of the sensors intelligently since the IEEE1451 protocol is embedded, and the system was implemented with CPLD and wireless communication applications. This can be used mostly in the IOT environments which contain a high-speed data acquisition system for the real-time cases. With the water environmental application in the IOT environment, the system was thus verified and achieved the good effective results in the practical application.

\section{The Aim and Objectives of the Study}

It could be concluded that most of the previous works focus on the monitoring systems and collecting the data in a database to store it, also in all of the above cases, it is noted that the (IOT) prove its efficiency day after day. However, this work will focus on another aspect of the (IOT), which is when proving the efficiency of the IOT and the need for increasing the number of applications, it must be noticed that these things need more addresses.

Therefore, we proposed a system that consists of many sensing nodes which will send their data to the server over only one IP address. By the implemented algorithm, one could decrease the number of needed IP addresses by implementing a gateway sink-node that has an IP address. This gateway collects the data from four sensing nodes using the low-cost NRF24 RF transceiver module. The collected data will be processed in the server and will be visualized using a Web-portal.

\section{$4 \quad$ Materials and Research Methods}

For the proposed system, the materials that were used are ESP 32, DHT 11 sensor, Nrf24L01, Arduino pro mini, router, and a laptop.

The DHT11 was used to detect the temperature and Humidity. The Arduino pro mini is used to processing the data then using Nrf214L01 via a non-IP Network to transmitting the data.to the sink node.

The ESP32 is used to collect the data from four sensor nodes and processing the collected data to be ready to transmitting it to the server by using the Router via a WIFI connection.

The server will collect the data from 50 sink nodes and process the collected data to be ready for visualization in a WEP-Portal. By this scenario, the data rate was decreased due to using a Non-IP network in many Sensor Nodes, In another words, there are 50 sink node. Each sink node collects data from 4 sensor nodes. Then, there are 200 sensor nodes. These sensor nodes transmit there data by using a Non-IP network. the data rate that needed for transmit the same amount of data in IOT applications will decreased because that the two hundred sensor nodes will not need to transmit the information of the IP address, the MAC address, and the port address. 


\section{System Model}

The proposed system is consisting of three parts: Sensor Nodes part, Sink Nodes part, and Server part. Each sensor node consists of five main parts which are: power supply, Microcontroller, Sensor, memory, and transmitter. As explained in figure 1.

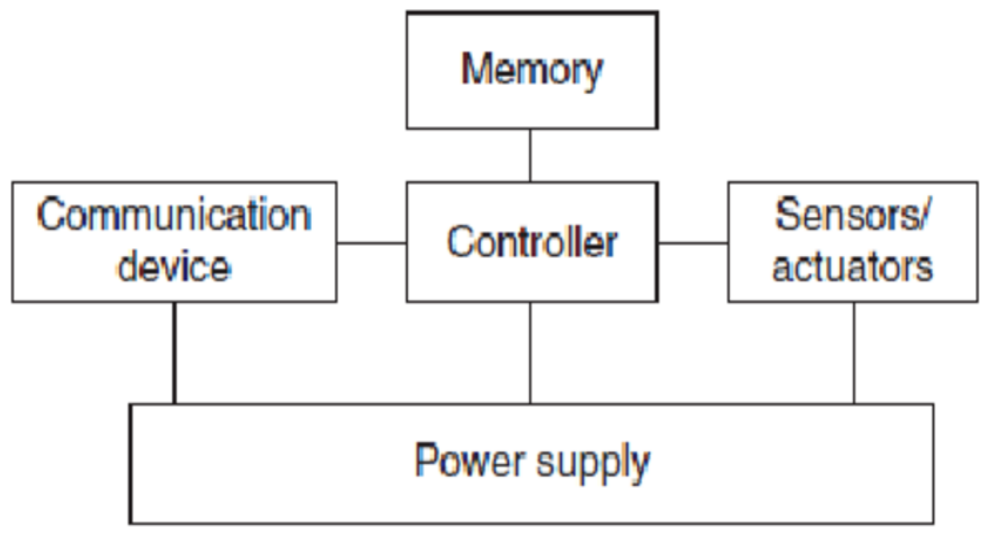

Fig. 1. Basic Sensor Node Components

The power supply for sensor nodes, the communication device, and the microcontroller that were used are adapter 5V, NRF24L01, Arduino pro mini respectively. The memory is always integrated with the Arduino pro mini, and the sensor that was used is the DHT11 which is sense temperature and humidity. Figure 2 explains the connection circuit of the sensor node.



Fig. 2. The connection circuit of the sensor node. 
The embedded system software is written in Arduino C programming language using the Arduino IDE Studio for development. Figure 3 is a flowchart to describe the parts of software.

It can be noticed that each sensing node has three steps after start as explained in

The first step is for configuring the transmitter module to be matched to the designed network. The transmitter will be configured by setting its transmitting channel, data rate, transmitting pipes, and transition power. All sensor nodes are configured to set the same transmit channel (which is channel number 7), the same data rate (which is $250 \mathrm{Kbps}$ ), and the same transmitting power (which is at $12 \mathrm{dbm}$ ).

But, the transmitting pipes must be differed from a node to another. Each sensor node must have a unique transmitting pipe because it will specify the sensor node address in the network.

After completing the configuring, the sensor node will be ready to receive data from the connected sensor. The received data must be processed before transmitting. The processing of the data is necessary to let the sink node knows how to handle with the received data. This happens by putting the comma symbol "," after each valid information. Also, it is used to separating between any different values from the sensors that providing more than one value. Because in the monitoring system, one needs to let all sensors data arrive to the server at the same time.

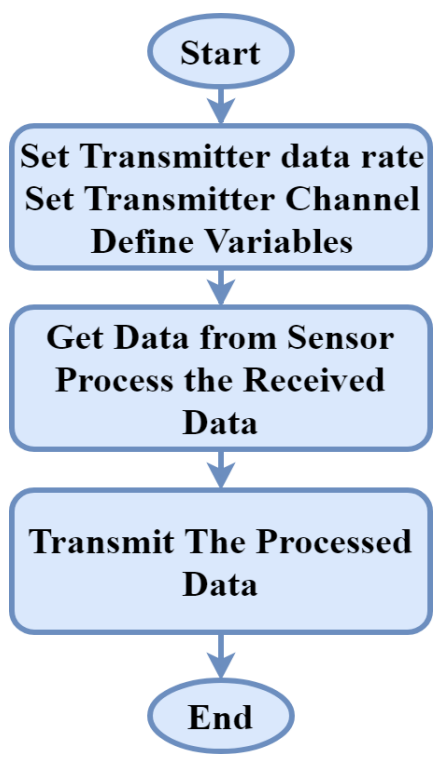

Fig. 3. The sensor nodes algorithm.

The sink-node is used to forwarding the data from sensors to the server, the connection circuit of the sink node is clarified in figure (4).

Also the software part of the proposed sink node has been written by Arduino $\mathrm{C}$ programming language through using the IDE studio platform. The proposed software algorithm was explained in figure (5) below. 


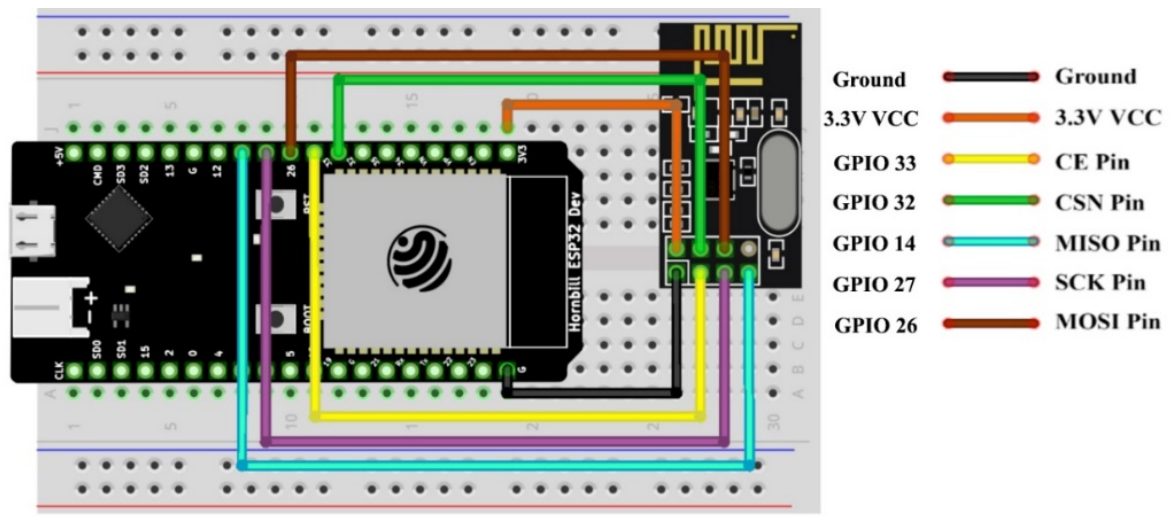

Fig. 4. The circuit diagram of the proposed sink node.

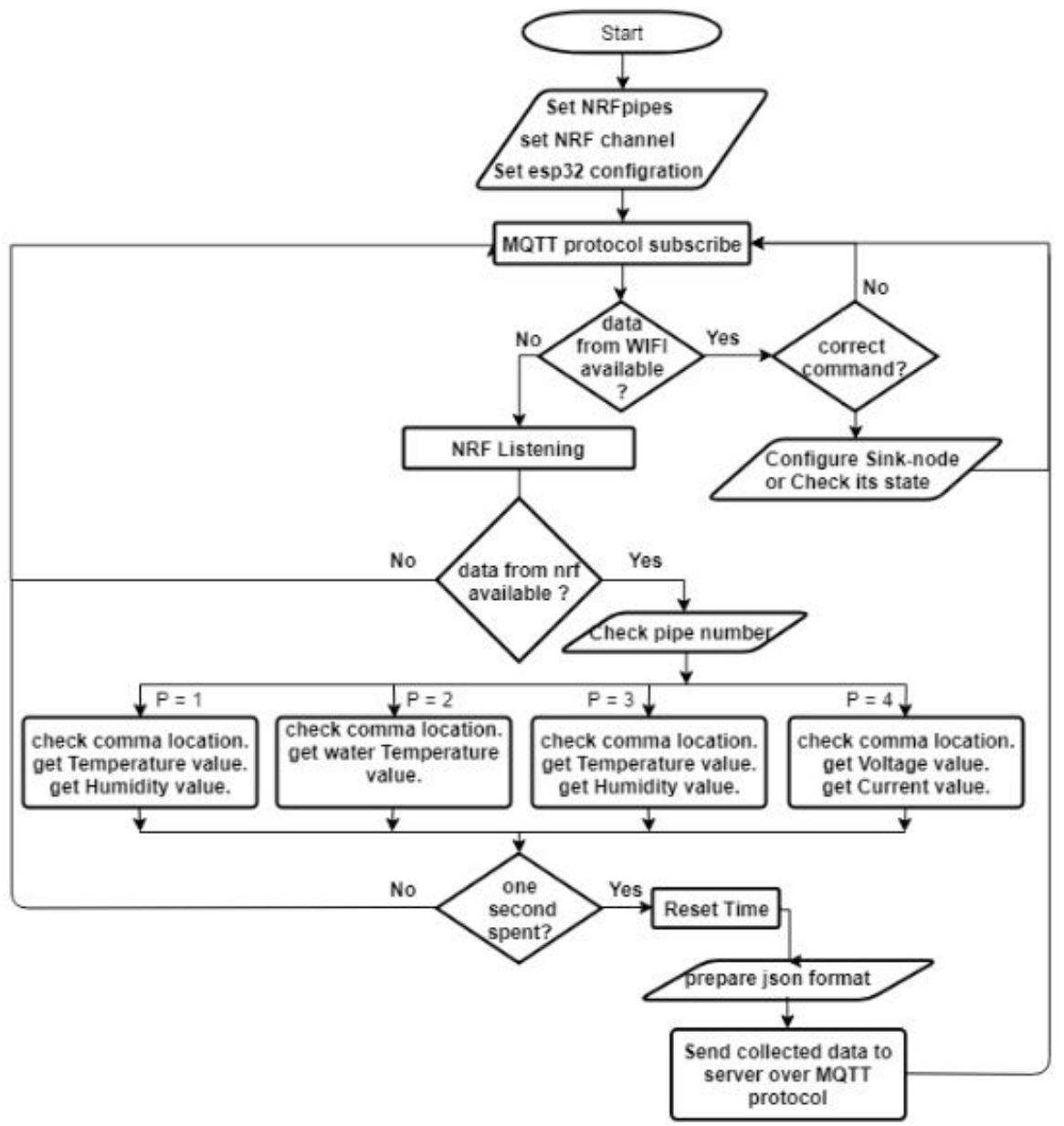

Fig. 5. The proposed algorithm of the sink node. 
Finally, the last part of the proposed system is the server, the server is a Laptop that use a Linux Operating system and developed in it a WEP-Portal that is able to visualize the collected and processed data.

\section{$6 \quad$ Results}

As explained, there are two networks in this system; the first network is between the sensor nodes and the sink node, while the second network is between the sinknode and the server. The first one as explained does not use IP address; it depends on the non-IP network by using the nRF24L01 modules. However, the second one uses IP network. By using the proposed system design, It can be noticed that the transmission in the proposed design is transmitting bits per second less than the systems that depend on the IP network only, as will explained in table (1).

By calculating the amount of bytes that are transmitted via IP network when used the DHT11 Sensor, each sensor node will transmit 47 byte/s. While when using the proposed system that it is depending on Non-IP Network, each sensor node will transmit 13 byte/s. Depending on the above optioned results, the below comparison shows the difference in transmitting rate between the proposed system and the general system.

Table 1. Comparison of the data transmission rate between the proposed system and the general system.

\begin{tabular}{|c|c|c|}
\hline Sensor Node & $\begin{array}{c}\text { Data Transmission rate in Non-IP } \\
\text { Network }\end{array}$ & $\begin{array}{c}\text { Data Transmission rate in IP } \\
\text { Network }\end{array}$ \\
\hline Temperature and Humidity & $200 \times 13=325$ byte/s & $200 \times 47=9400$ byte/s \\
\hline
\end{tabular}

One of the important applications that use WSN to collect the data in IOT systems is the Macao Meteorological and Geophysical Bureau that provides the service of monitoring the quality of the air. This application consists of many and different weather stations around the island. However, a very little number of regions were covered in this application. The proposed design can serve as complementary design to the existing system, by using more sensor nodes and depending on the multi-hop Architecture. The distribution of the sensor nodes can be in a much wider map.

\section{Conclusion}

From the results that obtained from the proposed design and implementation of the wireless sensor network for IOT application based on non-IP network, the main concluding remarks can be summarized as follows:

- The proposed design performed an IOT application for a monitoring system depends on the non-IP network.

- The proposed system approves that the data will be reduced by using the non-IP networks in WSN for IOT applications. 


\section{Acknowledgement}

This work is partly supported by Northern technical University (NTU), Mosul, Iraq, Wasit University College of Engineering, Wasit, Iraq, and University of Bridgeport (UB), Bridgeport, CT, USA.

\section{$9 \quad$ References}

[1] G. H. Kumar, G. Ramesh, C. J. I. J. o. P. Avadi, and A. Mathematics, "Novel gateway free device to device communication technique for IoT to enable direct communication between homogeneous devcies," vol. 118, no. 16, pp. 565-578, 2018.

[2] F. Huet and V. Boitier, "Design Strategy of Conventional Electronic for Wireless Sensor Node Powered by Vibration Energy Harvester," 2019. https://doi.org/10.2 4084/repqj17.375

[3] C. Lin, Z. Shang, W. Du, J. Ren, L. Wang, and G. Wu, "CoDoC: A Novel Attack for Wireless Rechargeable Sensor Networks through Denial of Charge," in IEEE INFOCOM 2019IEEE Conference on Computer Communications, 2019, pp. 856-864: IEEE. https://doi.org/10.1109/infocom.2019.8737403

[4] P. Pawar and A. J. I. T. R. Trivedi, "Device-to-device communication based IoT system: benefits and challenges," vol. 36, no. 4, pp. 362-374, 2019. https://doi.org/10.1080 /02564602.2018.1476191

[5] S. M. Bhat and S. Ahmed, "Internet of Things: An Insight into Emerging Applications and Architectures," 2018.

[6] H. Alrikabi, A. H. Alaidi, and K. J. I. J. o. I. M. T. Nasser, "The Application of Wireless Communication in IOT for Saving Electrical Energy," vol. 14, no. 01, pp. 152-160, 2020. https://doi.org/10.3991/ijim.v14i01.11538

[7] Z. Sheng, C. Mahapatra, C. Zhu, and V. C. J. I. a. Leung, "Recent advances in industrial wireless sensor networks toward efficient management in IoT," vol. 3, pp. 622-637, 2015. https://doi.org/10.1109/access.2015.2435000

[8] R. Rajesh, C. Annadurai, and K. J. C. C. Nirmaladevi, "Performance enhancement of IPv6 low power wireless personal area networks (6LoWPAN) by Lamport's algorithm," pp. 1-6, 2017. https://doi.org/10.1007/s10586-017-1324-3

[9] A. Ed-daoudy and K. J. J. o. B. D. Maalmi, "A new Internet of Things architecture for realtime prediction of various diseases using machine learning on big data environment," vol. 6, no. 1, p. 104, 2019. https://doi.org/10.1186/s40537-019-0271-7

[10] H. T. Alrikabi, A. H. M. Alaidi, A. S. Abdalrada, and F. T. J. I. J. o. E. T. i. L. Abed, "Analysis the Efficient Energy Prediction for 5G Wireless Communication Technologies," vol. 14, no. 08, pp. 23-37, 2019. https://doi.org/10.3991/ijet.v14i08.10485

[11] B. Song, H. Choi, and H. S. Lee, "Surveillance tracking system using passive infrared motion sensors in wireless sensor network," in 2008 International Conference on Information Networking, 2008, pp. 1-5: IEEE. https://doi.org/10.1109/icoin.2008.4472790

[12] Y. Zhai and X. Cheng, "Design of smart home remote monitoring system based on embedded system," in 2011 IEEE 2nd International Conference on Computing, Control and Industrial Engineering, 2011, vol. 2, pp. 41-44: IEEE. https://doi.org/10.1109 /ccieng.2011.6008062 
[13] A. Aggarwal and R. Joshi, "WSN and gsm based remote home security system," in International Conference on Recent Advances and Future Trends in Information Technology, iRAFIT, 2012.

[14] D. M. Holstius, A. Pillarisetti, K. Smith, and E. J. A. M. T. Seto, "Field calibrations of a low-cost aerosol sensor at a regulatory monitoring site in California," vol. 7, no. 4, pp. 1121-1131, 2014. https://doi.org/10.5194/amt-7-1121-2014

[15] J. Kim, J. Byun, D. Jeong, M.-i. Choi, B. Kang, and S. J. I. J. o. D. S. N. Park, "An IoTbased home energy management system over dynamic home area networks," vol. 11, no. 10, p. 828023, 2015. https://doi.org/10.1155/2015/828023

[16] T. T. Rita and Y. Xiao, "A portable Wireless Sensor Network system for real-time environmental monitoring," in 2016 IEEE 17th International Symposium on A World of Wireless, Mobile and Multimedia Networks (WoWMoM), 2016, pp. 1-6: IEEE. https://doi.org/10.1109/wowmom.2016.7523588

[17] J. M. Talavera et al., "Review of IoT applications in agro-industrial and environmental fields," vol. 142, pp. 283-297, 2017.

[18] H. T. S. ALRikabi, A. H. M. Alaidi, and F. T. Abed, "Attendance System Design And Implementation Based On Radio Frequency Identification (RFID) And Arduino."

[19] G. Sridevi, S. Satyanarayana, and P. S. Kumar, "Implementing the Reconfigurable Intelligent Sensor Interface in Wireless Networks," in Proceedings of 2nd International Conference on Micro-Electronics, Electromagnetics and Telecommunications, 2018, pp. 629-636: Springer. https://doi.org/10.1007/978-981-10-4280-5 66

\section{Authors}

Omar Hashim Yahya, Lecturer, Northern Technical University, Engineering Technical College of Mosul, Department of Computer Technology Engineering. Northern technical university, Mosul, Iraq The number of articles in national databases: 1. The number of articles in international databases: 4. Email: Omer h yahya@,ntu.edu.iq. Contact: - +9647722118786.

Haider Th. Salim ALRikabi, He is presently one of the faculty college of engineering, electrical engineering department, Wasit University in Al Kut, Wasit, Iraq. He received his B.Sc. degree in Electrical Engineering in 2006 from the Al Mustansiriya University in Baghdad, Iraq. his M.Sc. degree in Electrical Engineering focusing on Communications Systems from California state university/Fullerton, USA in 2014 .His current research interests include Communications systems with mobile generation, Control systems, intelligent technologies, smart cities, and Internet of Things (IoT) .Al Kut city - Hay ALRabee, Wasit, Iraq. The number of articles in national databases -8 . The number of articles in international databases -10 . Email:- hdhiyab@uowasit.edu.iq.Contact: - +9647725273435.

Ibtisam A. Aljazaery, College Of Engineering / Electronics. Engineering. Department, Babylon University, Hillah, Iraq. E-mail:- ibtisamalasady@gmail.com.

Article submitted 2020-01-04. Resubmitted 2020-02-12. Final acceptance 2020-02-17. Final version published as submitted by the authors. 